\title{
Moebius Syndrome
}

National Institute of Neurological Disorders and Stroke (NINDS)

\section{Source}

National Institute of Neurological Disorders and Stroke (NINDS). Moebius Syndrome

Information Page.

Moebius syndrome is a rare birth defect caused by the absence or underdevelopment of the 6th and 7th cranial nerves, which control eye movements and facial expression. Many of the other cranial nerves may also be affected, including the 3rd, 5th, 8th, 9th, 11th and 12th. The first symptom, present at birth, is an inability to suck. Other symptoms can include: feeding, swallowing, and choking problems; excessive drooling; crossed eyes; lack of facial expression; inability to smile; eye sensitivity; motor delays; high or cleft palate; hearing problems and speech difficulties. Children with Moebius syndrome are unable to move their eyes back and forth. Decreased numbers of muscle fibers have been reported. Deformities of the tongue, jaw, and limbs, such as clubfoot and missing or webbed fingers, may also occur. As children get older, lack of facial expression and inability to smile become the dominant visible symptoms. Approximately 30 to 40 percent of children with Moebius syndrome have some degree of autism.

There are four recognized categories of Moebius syndrome:

- Group I, characterized by small or absent brain stem nuclei that control the cranial nerves;

- Group II, characterized by loss and degeneration of neurons in the facial peripheral nerve;

- Group III, characterized by loss and degeneration of neurons and other brain cells, microscopic areas of damage, and hardened tissue in the brainstem nuclei, and,

- Group IV, characterized by muscular symptoms in spite of a lack of lesions in the cranial nerve. 